\title{
Genotypic analysis of nontuberculous mycobacteria isolated from raw milk and human cases in Wisconsin
}

\author{
Zeinab I. Ali, ${ }^{1 *} \odot$ Mostafa Hanafy, ${ }^{2,3 *}$ ๑ Chungyi Hansen, ${ }^{2}$ Adel M. Saudi, ${ }^{1}$ and Adel M. Talaat ${ }^{2} \dagger \oplus$ \\ ${ }^{1}$ Department of Food Hygiene and Control, Faculty of Veterinary Medicine, Cairo University, Giza, 12211, Egypt \\ ${ }^{2}$ Department of Pathobiological Sciences, University of Wisconsin, Madison 53706 \\ ${ }^{3}$ Department of Microbiology and Immunology, Faculty of Veterinary Medicine, Cairo University, Giza, 12211, Egypt
}

\begin{abstract}
Nontuberculous mycobacteria (NTM) compose a group of mycobacteria that do not belong to the Mycobacterium tuberculosis complex group. They are frequently isolated from environmental samples such as water, soil, and, to a lesser extent, food samples. Isolates of NTM represent a major health threat to humans worldwide, especially those who have asthma or are immunocompromised. Human disease is acquired from environmental exposures and through consumption of NTM-contaminated food. The most common clinical manifestation of NTM disease in human is lung disease, but lymphatic, skin and soft tissue, and disseminated disease are also important. The main objective of the current study was to profile the farm-level contamination of cow milk with NTM by examining milk filters and bulk tank milk samples. Five different NTM species were isolated in one dairy herd in Wisconsin, with confirmed 16S rRNA genotypes including Mycobacterium fortuitum, Mycobacterium avium ssp. hominissuis, Mycobacterium abscessus, Mycobacterium simiae, and Mycobacterium avium ssp. paratuberculosis (Mycobacterium paratuberculosis). In tank milk samples, $M$. fortuitum was the predominant species in $48 \%$ of the samples, whereas M. chelonae/abscessus and M. fortuitum were the only 2 species obtained from 77 and $23 \%$ of the examined filters, respectively. Surprisingly, M. avium ssp. hominissuis, M. paratuberculosis, and M. simiae were isolated from 16.7, 10.4, and $4 \%$ of the examined milk samples, respectively, but not from milk filters. Interestingly, NTM isolates from human clinical cases in Wisconsin clustered very closely with those from milk samples. These findings suggest that the problem of NTM contamination is underestimated in dairy herds and could contribute to human infections
\end{abstract}

Received January 17, 2020.

Accepted August 17, 2020.

*These authors contributed equally to this work.

†Corresponding author: Adel.Talaat@wisc.edu with NTM. Overall, the study validates the use of bulk tank samples rather than milk filters to assess contamination of milk with NTM. Nontuberculous mycobacteria represent one type of pathogens that extensively contaminate raw milk at the farm level. The significance of our research is in evaluating the existence of NTM at the farm level and identifying a simple approach to examine the potential milk contamination with NTM members using tank milk or milk filters from dairy operations. In addition, we attempted to examine the potential link between NTM isolates found in the farm to those circulating in humans in Wisconsin.

Key words: nontuberculous mycobacteria, cow tank milk, milk filter, human cases

\section{INTRODUCTION}

Nontuberculous mycobacteria (NTM), also known as environmental mycobacteria due to their abundant existence in the environment, are opportunistic pathogens of public health concern, especially for immunocompromised individuals (Claeys and Robinson, 2018). Members of NTM are the causative agents of many human infections such as lymphadenitis in children and pulmonary, skin, and soft tissue infections (Nishiuchi et al., 2017). Although human-to-human transmission is rare, it is essential to identify potential sources and routes of exposure to NTM. Although the risk of NTM transmission through water and several foods is documented (Argueta et al., 2000), the prevalence and risk of NTM in animal products, such as milk, is understudied. Globally, NTM isolates have been detected in milk products from both high- and low-income countries (Yoo et al., 2012; Kendall and Winthrop, 2013; Panagiotou et al., 2014). Nontuberculous mycobacteria are considered emerging milk-borne pathogens worldwide. In Brazil, $21.7 \%$ of mozzarella cheese manufactured from raw buffalo milk was positive for NTM, demonstrating a risk for consumers (Jordão Junior et al., 2009). Another study, in Spain, reported the presence of viable Mycobacterium avium ssp. hominissuis 
in a powdered infant formula and mycobacterial DNA (M. avium, Mycobacterium tuberculosis complex, and other NTM mycobacteria) was detected in $15 \%$ of the examined dairy products (Sevilla et al., 2017). Finally, Mycobacterium gordonae, Mycobacterium fortuitum, Mycobacterium senegalense, and $M$. avium have been isolated from fresh milk sold in Nigeria, suggesting a high prevalence of NTM in dairy food (Agada et al., 2014). In Taiwan, the incidence of NTM infections increased from 2.7 to 10.2 cases per 100,000 between the years 2000 and 2008 (Lai et al., 2010). The incidence of NTM infection increased from 0.9 per 100,000 population in 1995 to 2.9 per 100,000 in 2006 in England, Wales, and Northern Ireland (Moore et al., 2010). The increase in NTM prevalence highlights the need to examine potential sources of infection and develop protocols to eliminate them. With the increasing popularity of raw milk consumption throughout the United States (allowed in 30 of 50 states; Costard et al., 2017), surveillance of NTM in milk and dairy herds is becoming of a paramount importance. This study was designed to help close this knowledge gap.

Previously, NTM have been detected in meat and cow milk (raw and pasteurized; Sgarioni et al., 2014), major food sources for humans. Water, soil, and food have been suggested as important NTM sources for infection transmission to humans (Shitaye et al., 2009; Sgarioni et al., 2014). The relationship between environmental sources of NTM and disease in humans has been studied for decades (Wolinsky, 1979), but mostly as association studies because of a lack of data on the genetic basis of the relationship. However, with the advancement of molecular techniques, several reports have suggested a stronger link between NTM in food samples and those circulating in patients (Yoder et al., 1999). In California, 25 isolates of NTM, including $M$. avium, M. gordonae, and Mycobacterium simiae, were recovered from 121 supermarket food items, mainly of plant origin (Argueta et al., 2000). Unfortunately, the published data regarding the prevalence of NTM in food of animal origin are scarce in the United States. In Brazil, NTM was found in 9 to $25 \%$ of raw milk samples and related dairy products (Franco et al., 2013). Similar levels were obtained from analyses of raw and processed meat samples from the Czech Republic (Shitaye et al., 2009). High levels of NTM contamination were also reported in raw milk samples analyzed in several countries from Europe and Africa (Kazwala et al., 1998; Konuk et al., 2007). Both PCR and genetic fingerprinting-based approaches (e.g., variable number of tandem repeats, DNA sequencing) were suggested to accelerate the detection of the prevalence and persistence of NTM in food samples (Bolanos et al., 2017).
Here, we hypothesized that the diversity of NTM contamination is underestimated in dairy products, which should be of concern to public health authorities. Our study was conducted to develop a simple approach to examine the potential milk contamination with NTM on the farm level using tank milk and milk filters from dairy operations. In addition, we estimated the level of diversity among NTM present in raw milk and examined any potential link to the NTM isolated from patients in Wisconsin.

\section{MATERIALS AND METHODS}

\section{Milk Sample Collection}

A medium-size local dairy herd with 80 lactating cows was used for this investigation from January to May 2016. Milk filters and raw milk samples were collected from the bulk tank with a total of 85 samples for each (milk filters and tank milk samples $=170$ samples). Milk filters are usually placed just before the bulk tank to remove gross foreign material from milk. The 85 raw milk samples $(50 \mathrm{~mL}$ each) were collected using aseptic technique directly from the bulk tank that contains the milk that has passed through the milk filter. The milk filters and tank milk samples were maintained at $4^{\circ} \mathrm{C}$ during transport and prepared as described before (Slana et al., 2012). Briefly, each filter was placed separately in a stomacher bag with $100 \mathrm{~mL}$ of PBS (pH 7.0) containing $0.05 \%$ Tween 20 (PBS-T) and homogenized in a stomacher for $2 \mathrm{~min}$. Sample homogenates were centrifuged (15 min at 2,500 $\times g$ ) and pellets were resuspended in $10 \mathrm{~mL}$ of PBS-T for culturing.

\section{Isolation of Mycobacteria from Filters and Milk Samples}

Ten milliliters from milk samples and $1 \mathrm{~mL}$ from the processed filter homogenates were centrifuged (15 min at $2,500 \times g)$ before the decontamination step. The obtained pellets and cream layers were resuspended in $10 \mathrm{~mL}$ of $0.75 \%$ 1-hexadecylpyridinium chloride (Sigma-Aldrich, St. Louis, MO) and incubated for 5 $\mathrm{h}$ at room temperature for decontamination. Samples were centrifuged again $(15$ min at 2,500 $\times g$ ) and pellets were resuspended in $1 \mathrm{~mL}$ of PBS for inoculation onto Lowenstein Jensen (LJ) medium (LJ slants) and Middlebrook 7H10 agar (BD, Franklin Lakes, NJ) as described before (Dundee et al., 2001). Cultures were incubated in the presence of 5 to $10 \% \mathrm{CO}_{2}$ at $37^{\circ} \mathrm{C}$ for $90 \mathrm{~d}$ and inspected weekly for bacterial growth (Ghosh et al., 2012). One set of Middlebrook 7H10 plates was 
supplemented with mycobactin $\mathbf{J}$ (MJ, $2 \mathrm{mg} / \mathrm{mL}$ ), an iron-chelating cell wall component for the isolation of M. paratuberculosis, and $2 \mu \mathrm{g}$ of vancomycin, $30 \mu \mathrm{g}$ of amphotericin B, and $20 \mu \mathrm{g}$ of nalidixic acid (VAN) per milliliter. All antibiotics were from Sigma Chemical Co. (St. Louis, MO) Colonies growing on Mycobacteriumspecific media were characterized by Ziehl-Neelsen staining and 16S rRNA PCR. For Ziehl-Neelsen staining, wet smears from each suspected colony were prepared on a clean and dry glass slide and heat fixed over flame until dried. Slides were placed on a $90^{\circ} \mathrm{C}$ adjusted heat block, and carbol-fuchsin solution was added. After 5 min, slides were washed with distilled water, followed by an additional wash with $1 \%$ acid alcohol for discoloration. The slides were counterstained with methylene blue solution for $30 \mathrm{~s}$ and washed with distilled water before air drying and microscopical examination.

\section{NTM Isolates from Human Cases}

With the help of Wisconsin State Laboratory of Hygiene (Madison), we obtained 13 de-identified isolates of NTM that were collected from human patients with diagnosed NTM infections cultured from various sources including sputum, urine, blood, or neck tissues. All isolates were cultured as detailed above and their DNA was extracted for PCR amplification as detailed below.

\section{Extraction of Genomic DNA}

To obtain high-quality genomic DNA (gDNA), we followed a method previously developed in our group (Talaat et al., 1997). Briefly, mycobacterial cultures were pelleted by centrifugation, and the pellets were washed and resuspended in equal volume of Tris-EDTA buffer. Bacterial suspensions were placed at $80^{\circ} \mathrm{C}$ for 20 min to kill all living organisms. Tubes were allowed to cool at room temperature, and $10 \mu \mathrm{L}$ of $100 \mathrm{mg} /$ $\mathrm{mL}$ lysozyme was then added to each tube, followed by incubation at $37^{\circ} \mathrm{C}$ for $3 \mathrm{~h}$ with occasional mixing. A solution of $10 \%$ SDS and proteinase $\mathrm{K}(20 \mathrm{mg} / \mathrm{mL})$ was added to each tube at a ratio of 88:12. Tubes were incubated at $65^{\circ} \mathrm{C}$ for $2 \mathrm{~h}$ followed by addition of 100 $\mu \mathrm{L}$ of $5 \mathrm{M} \mathrm{NaCl}$ at $65^{\circ} \mathrm{C}$ for additional $10 \mathrm{~min}$. A $10 \%$ solution of cetyl trimethyl ammonium bromide $(80 \mu \mathrm{L}$; Sigma-Aldrich) was added, followed by mixing and incubation at $65^{\circ} \mathrm{C}$ for another $10 \mathrm{~min}$. Then, DNA was extracted with an equal volume of phenol:chloroform: isoamylalcohol (25:24:1 vol:vol:vol) and similarly with chloroform:isoamylalcohol (24:1 vol:vol) followed by precipitation with 0.6 volumes of ice-cold isopropanol and centrifugation $(14,000 \times g)$ at $4^{\circ} \mathrm{C}$ for $15 \mathrm{~min}$. Pellets were washed with cold $75 \%$ ethanol and dried in a SpeedVac (Thermo Scientific, Wilmington, DE) for $5 \mathrm{~min}$. The DNA was finally resuspended in $50 \mu \mathrm{L}$ of sterile distilled water. Quality of the gDNA was verified by both NanoDrop (Thermo Scientific) machine and electrophoresis. To detect the presence of mycobacterial DNA in the collected samples, gDNA was extracted directly following our previously described procedure (Talaat et al., 1997).

\section{Polymerase Chain Reaction}

To confirm the identity of the mycobacterial colonies and gDNA extracted directly from the examined samples, we subjected them to PCR amplification targeting the $16 \mathrm{~S}$ rRNA gene. A partial sequence (938 bp) of the 16S rRNA gene was amplified from all isolates and samples. Primers used for this purpose were designed by our group (Talaat et al., 1997); namely, AMT 36 (GCGAACGGGTGAGTAACACG) and AMT 37 (TGCACACAGGCCACAAGC). Each PCR amplification was $25 \mu \mathrm{L}$, which contained $1 M$ betaine, $50 \mathrm{~m} M$ potassium glutamate, $10 \mathrm{~m} M$ Tris- $\mathrm{HCl} \mathrm{pH} 8.8,0.1 \%$ Triton $\mathrm{X}-100,2 \mathrm{~m} M$ magnesium chloride, $0.2 \mathrm{~m} M$ dNTPs, 0.5 $\mathrm{m} M$ each primer, $0.5 \mathrm{U}$ of Taq DNA polymerase (Promega, Madison, WI), and $25 \mathrm{ng}$ of genomic DNA. The amplification cycle consisted of an initial denaturation step of $94^{\circ} \mathrm{C}$ for $5 \mathrm{~min}$; 35 cycles of denaturation at $94^{\circ} \mathrm{C}$ for $30 \mathrm{~s}$, annealing at $55^{\circ} \mathrm{C}$ for $30 \mathrm{~s}$, and extension at $72^{\circ} \mathrm{C}$ for $1 \mathrm{~min}$; and a final extension at $72^{\circ} \mathrm{C}$ for 7 min. Amplicons were evaluated by electrophoresis in $2 \%$ agarose gels prestained with ethidium bromide $(0.5$ $\mathrm{mg} / \mathrm{mL}$ ). Single band products of amplicons were purified from agarose gels and extracted using Wizard SV Gel and PCR cleanup system (Promega) as detailed before (Talaat et al., 1997).

\section{Sanger DNA Sequencing and Phylogenetic Analysis}

Following PCR amplification, purified PCR fragments were sequenced with BigDye Terminator v3.1 (Applied Biosystems, Foster City, CA) with AMT 36 and AMT 37 primers, according to the manufacturer's instruction. All sequences were analyzed with BLASTn algorithm on the NCBI web portal (http://blast.ncbi .nlm.nih.gov/Blast.cgi).

A rooted tree was computed using MEGA7 software (Kumar et al., 2016) and neighbor-joining method to align all sequences of $16 \mathrm{~S}$ rRNA and build a phylogenetic tree with the presence of reference sequences from several mycobacterial species deposited in GenBank (Tamura et al., 2013). 


\section{Whole-Genome Sequence Analysis and Phylogenetic Analysis}

Selected isolates from farm tank milk samples and de-identified human isolates from the Wisconsin State Laboratory of Hygiene were submitted to the Wisconsin Biotechnology Center for whole-genome sequencing (WGS) as detailed before by our group (Hsu et al., 2011; Abdelaal et al., 2019). Genomic libraries for a total of 19 isolates were prepared and run on IlluminaMiSeq 2000 platform (Illumina Inc., San Diego, CA). Reads with an average length of $250 \mathrm{bp}$ were assembled using SPAdes 3.14.1 (Bankevich et al., 2012) for de novo genome assembly. The assembled contigs were searched against the NCBI database using BLASTn (https:// blast.ncbi.nlm.nih.gov/Blast.cgi) to ensure the identity of each isolate. CLC Bio Workbench version 8.5.1 (https://digitalinsights.qiagen.com/) was used, and we used standard genome corresponding to identification from BLASTn; namely, M. avium 104 (accession number: CP000479.1), M. abscessus ATCC19977 (accession number: NC_010397.1), or M. fortuitum CT6 (accession number: CP011269.1), to identify SNP; insertions/deletions (InDels) event with minimum variation frequency was set to $50 \%$ to count SNP. Consensus sequences for mapped reads were exported from CLC Bio workbench version 8.5.1 as FASTA format and aligned using the Harvest software package (Treangen et al., 2014) and the "parsnp" function to output a newick tree file that was visualized using MEGA X software.

\section{Data Availability}

The sequences of the 16S rRNA gene have been deposited in the GenBank database under the accession numbers reported in Table 1. All whole-genome sequences for mycobacterial isolates used in this study were deposited at the NCBI GenBank (BioProject no. PRJNA656902) with BioSample numbers from SAMN15806900 to SAMN15806920. See Table 2 for isolate names, BioSample numbers, and isolate sources.

\section{RESULTS}

\section{Milk Contamination with NTM}

We hypothesized that examination of milk filters and tank milk would provide a means of detecting mycobacteria contamination on the herd level. Although milk filters trap debris, typically in the range of 100 to 150 $\mu \mathrm{m}$, they do not prevent pathogenic bacteria $(\sim 1-10$ $\mu \mathrm{m})$ from passing through. Culture-positive milk filters likely indicate that tank milk has been contaminated, but the reverse might not always be correct. Detecting
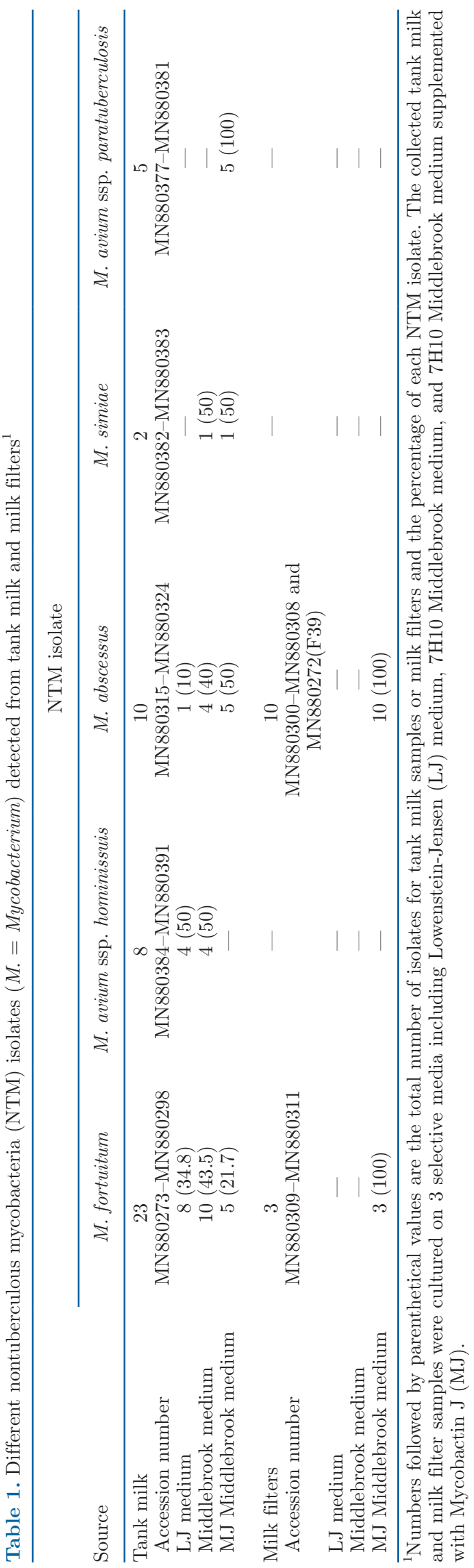


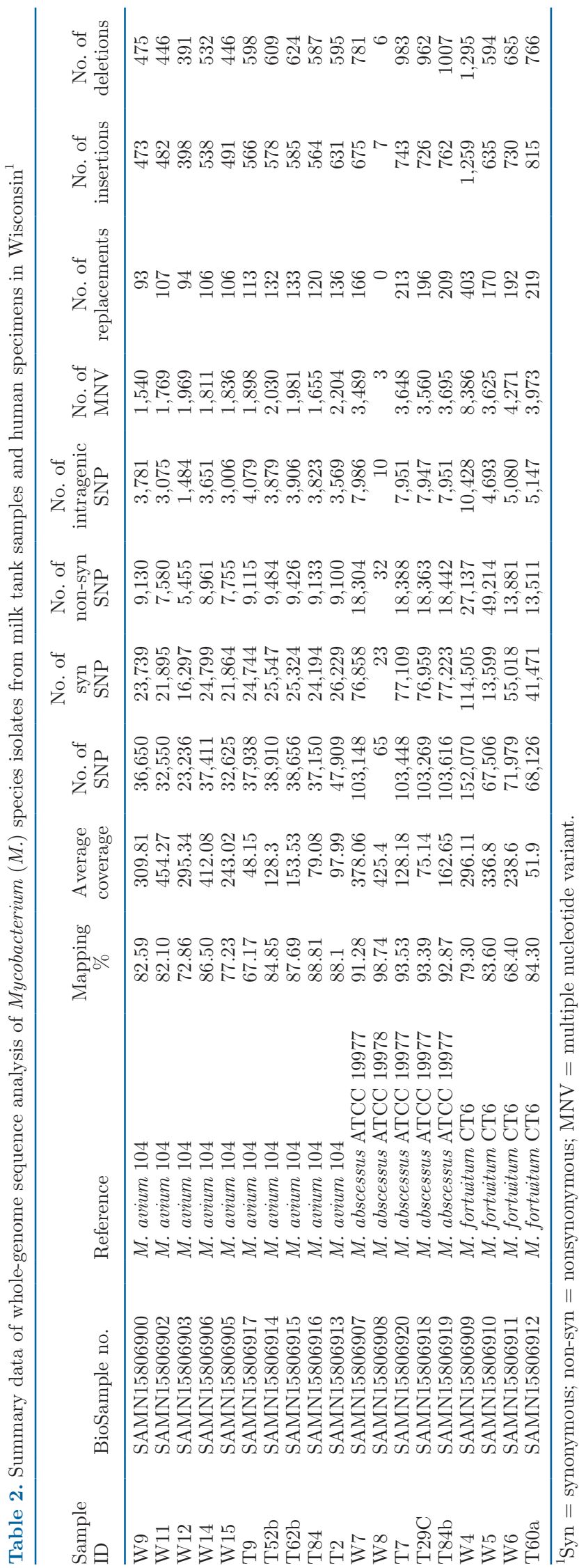

mycobacterial DNA directly from milk filters and the examined milk samples resulted in a total of 48 positive milk filter samples out of $85(56.5 \%)$ with almost the same percentage for the tank milk (44 samples out of $85,51.8 \%)$.

Using culturing on mycobacteria-specific media, tank milk and milk filter samples were positive in 17 (20\%) and $10(11.7 \%)$ samples, including 48 and 13 mycobacterial isolates (Table 1), respectively, with a total of 61 independent isolates cultured from the examined dairy herd.

A total of 5 mycobacterial species were isolated from the tank milk samples and only 2 were obtained from the milk filters (Table 1), an indication of the validity of sampling tank milk to assess herd health and milk contamination with NTM. All isolates grown on the selected media were positive for acid fast staining.

\section{Genotypes of NTM Present in Raw Milk}

To confirm isolate identity, we amplified the $16 \mathrm{~S}$ rRNA gene from all isolates. As expected, specific DNA bands of approximately $938 \mathrm{bp}$ were observed on agarose gel for all positive samples, indicating that all isolates were members of the genus Mycobacterium. To identify the species of mycobacterial isolates, amplicons from all isolates were sequenced using Sanger sequencing (Talaat et al., 1997). DNA sequences from all isolates were analyzed using the BLASTn (https: //blast.ncbi.nlm.nih.gov/Blast.cgi) algorithm against all bacterial genomes present in the GenBank database (NCBI Resource Coordinators, 2016). From the tank milk samples cultured on LJ slants, 13 independent isolates were grown. Among these isolates, 8 belonged to the $M$. fortuitum group, 4 belonged to $M$. avium ssp. hominissuis, and 1 was identified as M. abscessus (Table 1). The other set of tank milk samples was cultured on 7H10 medium, and 19 isolates were grown. Among them, M. fortuitum was the predominant species (n =10). Four isolates belonged to the $M$. avium ssp. hominissuis group, while 1 isolate was identified as $M$. abscessus and another was identified as M. simiae. In addition, when $7 \mathrm{H} 10$ medium was supplemented with MJ, 16 isolates were grown. Among them, 5 isolates belonged to each group for $M$. avium ssp. paratuberculosis, M. abscessus, and M. fortuitum, and 1 isolate was identified as M. simiae. For the milk filter samples, both LJ slants and 7H10 Middlebrook medium without antibiotics were heavily contaminated, a potential cause of isolation failure; however, all isolates were obtained from Middlebrook 7H10 medium supplemented with MJ and the antibiotics mixture (VAN). A total of 13 isolates were recovered from milk filters. Among them, 10 isolates belonged to the $M$. abscessus group, while 
the remaining 3 isolates belonged to the $M$. fortuitum group (Table 1).

\section{Phylogenetic Analysis of the NTM}

To better examine the relationship between NTM isolates, we examined the evolutionary distances among isolates based on 16S rRNA sequences. As expected, clear phylogenetic clusters formed between members of the $M$. abscessus/chelonae complex and the M. avium ssp. hominissuis/paratuberculosis complex. No significant genetic distance was found between members of the M. abscessus/chelonae complex whether they were isolated from tank milk or milk filters, suggesting a common source of contamination. All isolates of $M$. fortuitum occupied a different cluster between the other 2 clusters. As expected, 2 isolates of $M$. simiae clustered independently, another confirmation of their identity and potential different source of transmission. However, the low bootstrapping percentage $(<50 \%)$

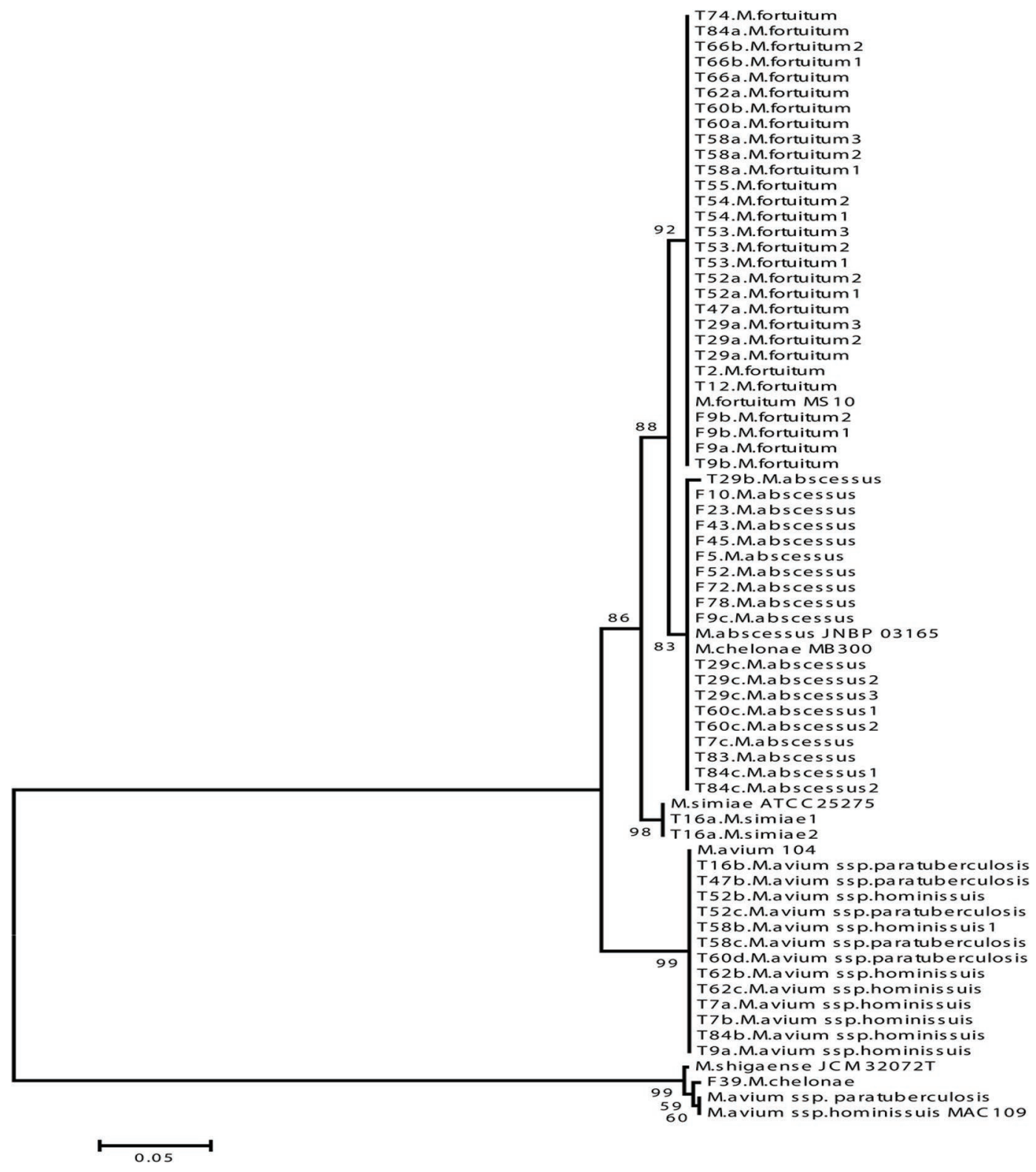

Figure 1. Evolutionary tree of all nontuberculous mycobacteria isolates from tank milk (identified as T samples) and milk filters (identified as F samples) isolated from a Wisconsin dairy herd. The tree is based on $16 \mathrm{~S}$ rRNA sequences using the neighbor-joining method in MEGA7 software (https://www.megasoftware.net/). The tree is drawn to scale with a bar representing the phylogenetic distance. Numbers next to the branches represent percentages of 1,000 bootstrap test. 
that distinguished the $M$. simiae from the $M$. avium/ paratuberculosis complex could indicate their evolutionary relatedness (Figure 1).

In another analysis, we tested the phylogenetic relatedness between selected farm NTM isolates $(\mathrm{n}=9)$ and those circulating in human patients $(\mathrm{n}=10)$ from Wisconsin, using de-identified samples from the collection of the Wisconsin State Laboratory of Hygiene. In Table 2 , we added a summary of the WGS of 19 NTM isolates from both milk and human samples. Single nucleotide polymorphisms for each isolate were compared with genomes representing the standard strains of $M$. avium ssp. hominissuis, M. fortuitum, and M. abscessus (Figure $2 \mathrm{~b})$. These isolates were the most commonly isolated mycobacteria from tank milk samples. Interestingly, the whole-genome sequences from all human isolates were clustered with isolates from milk samples, an indication of highly similar genotypes of NTM present in both milk samples and those circulating in humans infected with NTM (Figure 2). Moreover, the overall SNP 16S rRNA genotyping did not accurately identify 3 isolates of M. fortuitum (T2, T9, and T84), which WGS identified as M. abscessus for T2 and M. avium ssp. hominissuis for T9 and T84, respectively. On the other hand, $\mathrm{T} 52 \mathrm{~b}$ and $\mathrm{T} 62 \mathrm{~b}$ isolates that were identified by $16 \mathrm{~S}$ rRNA as $M$. avium ssp. hominissuis were identified by WGS as $M$. abscessus and $M$. fortuitum, respectively. This finding further confirms the superiority of WGS for strain identification compared with other standard genotyping approaches.

\section{DISCUSSION}

Nontuberculous mycobacteria are a group of opportunistic mycobacterial species that do not belong to the M. tuberculosis complex (Brode et al., 2017). These mycobacterial species have been isolated from many environmental sources, such as water, soil, and food (Nishiuchi et al., 2017), implicating these sources as vehicles for NTM transmission to humans (Sgarioni et al., 2014). Previously, analysis of raw milk and dairy products indicated the presence of high levels of NTM in some developing countries (Kazwala et al., 1998; Konuk et al., 2007). Unfortunately, in tuberculosisendemic countries (e.g., India, China), the problem of NTM is usually masked by problems associated with tuberculosis (Gopinath and Singh, 2010), suggesting an underestimation of NTM prevalence. Immunocompromised individuals are at high risk of contracting NTM infections, especially with the opportunistic members of the M. avium complex (Shojaei et al., 2011). The current study was conducted to examine the extent of NTM presence at the farm level, using a dairy herd in Wisconsin, where mycobacterial prevalence is low (Wis- consin Department of Health Services, 2019) compared with other locations (Gopinath and Singh, 2010). The milk filters are usually located just before the bulk tank and have pores of 65 to $75 \mu \mathrm{m}$ to filter debris and fecal material from the milk (McKee et al., 2002). Microbial analysis of milk filters represents a first-choice sampling strategy to assess the overall microbial quality of milk, hence we compared the utility of milk filters with that of tank milk samples to assess mycobacterial presence in unpasteurized milk. Unexpectedly, our analysis of both sampling strategies indicated the superiority of bulk tank milk sampling over milk filters in both the number of isolates and diversity of isolated species. This result is likely due to high level of contamination that could not be trapped by milk filters.

One of the major findings obtained in this study is that $M$. fortuitum was the predominate NTM isolate from tank milk as shown in Table 1, while $M$. chelonae/ abscessus was the prevailing isolate detected from milk filters. In an earlier study of NTM isolates, the M. avium complex, specifically $M$. avium ssp. paratuberculosis ( $M$. paratuberculosis), represented the highest prevalence, followed by $M$. fortuitum (Gopinath and Singh, 2010). In this report, M. paratuberculosis was isolated from 5 tank milk samples but was not detected at all from any of the milk filters, most likely because of its low level in the examined herd. Similar studies reported the presence of $M$. paratuberculosis in raw milk samples worldwide (Grant et al., 2002; Ayele et al., 2005; Slana et al., 2008; Botsaris et al., 2010; Shankar et al., 2010).

Related studies indicated the presence of NTM in both raw and pasteurized milk in Brazil including $M$. fortuitum, Mycobacterium marinum, Mycobacterium kansasii, and M. gordonae (Leite et al., 2003). In other studies, M. chelonae and Mycobacterium scrofulaceum were also isolated from raw and pasteurized milk (Sgarioni et al., 2014). In Turkey, other members of the NTM group were detected in raw milk including, Mycobacterium terrae, M. kansasii, Mycobacterium haemophilum, and Mycobacterium agri (Konuk et al., 2007). In our study, 5 NTM species involved in a variety of human illnesses were detected including $M$. fortuitum, $M$. chelonae/abscessus, M. paratuberculosis, M. avium ssp. hominissuis, and M. simiae.

To better identify each isolate, we sequenced a large proportion of the $16 \mathrm{~S}$ rRNA gene, which confirmed the identity of all mycobacterial isolates (Figure 1) and provided a tool for further analysis of such isolates in the context of other circulating mycobacterial species. Interestingly, phylogenetic analysis of milk isolate sequences and their counterparts from human cases of infection with NTM species circulating in Wisconsin patients indicated a close relationship between both groups of NTM (Figure 2) when whole genomes were 
analyzed. Such analysis suggested a potential common source of NTM isolates, most likely potable tap water as indicated before (Honda et al., 2018) for M. avium complex but not for $M$. paratuberculosis, M. abscessus, and $M$. fortuitum complexes. It is possible that animal infections with such organisms are transmissible to human through the consumption of raw milk or dairy products made with unpasteurized milk. Results presented here should prompt examination of more milk and dairy products for mycobacterial contamination, especially with the increase of consumption of raw milk throughout the United States (Costard et al., 2017) and in low-income countries as well. Moreover, contradictory results from $16 \mathrm{~S}$ rRNA genotyping and WGS of members of $M$. fortuitum and $M$. avium groups suggested the need to use more targets for genotyping (e.g., rpoB and hsp65) or the need to use WGS.

Overall, our study illustrates that examination of milk samples could help in the evaluation of mycobacterial diversity in a dairy herd, with bulk tank milk sampling being the superior strategy. The study validates the use of bulk tank samples over milk filters to assay milk contamination with NTM. Moreover, consumption of raw milk or unpasteurized dairy products constitutes a potential risk factor for contracting NTM infections in humans. The implementation of control measures that

a)

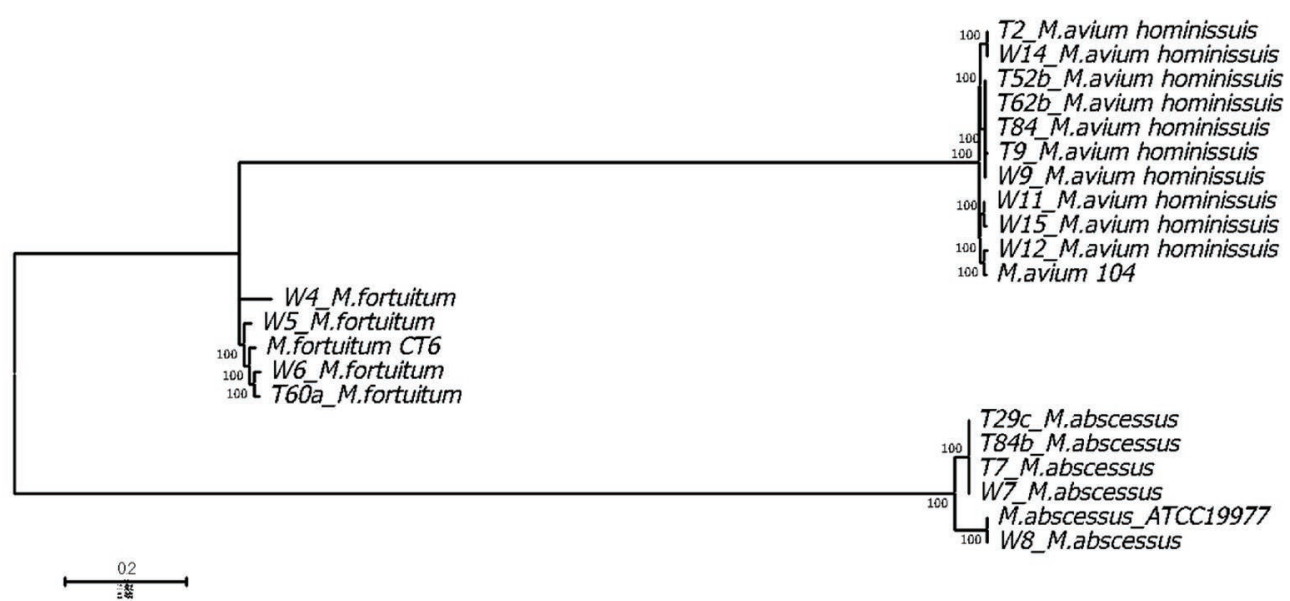

b)

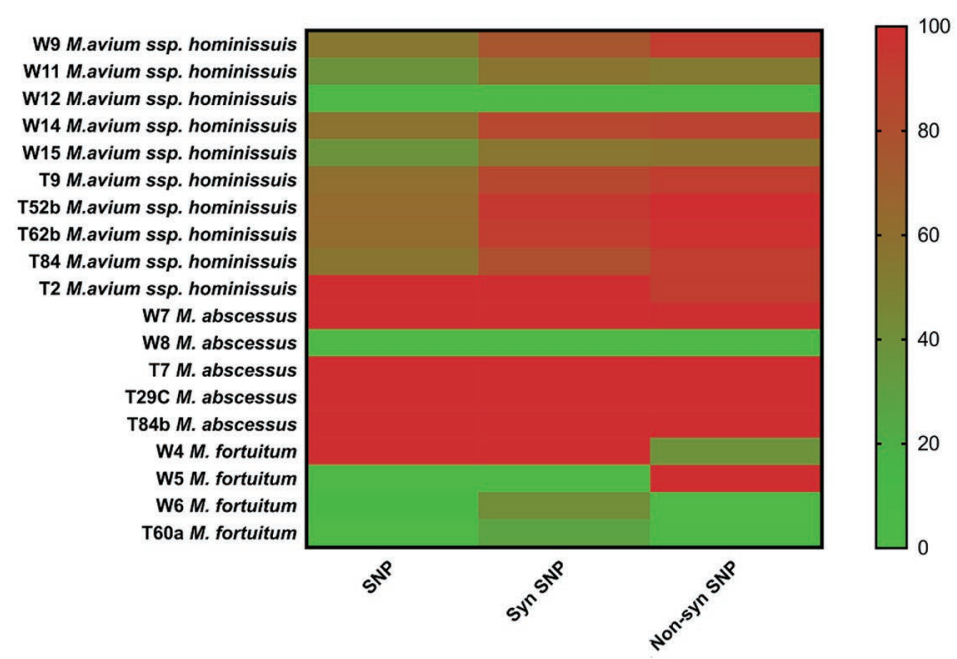

Figure 2. Evolutionary analysis of mycobacterial isolates from tank milk samples and human specimens. (a) Evolutionary tree based on whole-genome sequence analysis of 22 nontuberculous mycobacteria (NTM) isolates representing all groups detected in a Wisconsin dairy herd (designated with T) and human isolates from Wisconsin patients (designated with W). The unrooted tree is based on whole-genome sequences using Maximum Unique Match algorithm in the Harvest package and visualized by MEGA X software (https://www.megasoftware.net/). The nodes represent unique matches between bacterial genomes. The value on each branch is the posterior probability, showing the percentage support for the following node. (b) A heat map of NTM isolates displaying percentage of the total SNPs with synonymous (Syn) and nonsynonymous (Non-Syn) polymorphism when each isolate is compared with its own standard strain. The heat map was generated using GraphPad Prism version 7 (GraphPad Software Inc., La Jolla, CA). 
reduce or prevent contamination of raw milk with NTM during and after milking is of a paramount importance to avoid human illness associated with NTM infection.

\section{ACKNOWLEDGMENTS}

We also thank David Warshauer from the Wisconsin State Laboratory of Hygiene (Madison) for providing the NTM isolates from human cases. This work was partially supported by a USDA (Washington, DC) grant (NIFA-2018-67015-28243), United States Agency for International Development and National Academy of Sciences subaward no. 2000010565, and animal formula fund (no. WIS02015) awarded to AT. Any findings, conclusions, or recommendations expressed in this article are those of the authors alone, and do not necessarily reflect the views of USAID or NAS. In addition, Egyptian Culture and Educational Bureau Awards were made to ZA and $\mathrm{MH}$. The authors have not stated any conflicts of interest.

\section{REFERENCES}

Abdelaal, H. F. M., D. Spalink, A. Amer, H. Steinberg, E. A. Hashish, E. A. Nasr, and A. M. Talaat. 2019. Genomic polymorphism associated with the emergence of virulent isolates of Mycobacterium bovis in the Nile Delta. Sci. Rep. 9:11657. https://doi.org/10.1038/ s41598-019-48106-3.

Agada, C. A., H. K. Adesokan, D. Igwe, and S. I. Cadmus. 2014. Mycobacterium africanum and nontuberculous mycobacteria from fresh milk of pastoral cattle and soft cheese in Oyo State-implications for public health. Afr. J. Med. Med. Sci. 43(Suppl):13-20.

Argueta, C., S. Yoder, A. E. Holtzman, T. W. Aronson, N. Glover, O. G. Berlin, G. N. Stelma Jr., S. Froman, and P. Tomasek. 2000. Isolation and identification of nontuberculous mycobacteria from foods as possible exposure sources. J. Food Prot. 63:930-933. https://doi.org/10.4315/0362-028X-63.7.930.

Ayele, W. Y., P. Svastova, P. Roubal, M. Bartos, and I. Pavlik. 2005. Mycobacterium avium subspecies paratuberculosis cultured from locally and commercially pasteurized cow's milk in the Czech Republic. Appl. Environ. Microbiol. 71:1210-1214. https://doi.org/ 10.1128/AEM.71.3.1210-1214.2005.

Bankevich, A., S. Nurk, D. Antipov, A. A. Gurevich, M. Dvorkin, A. S. Kulikov, V. M. Lesin, S. I. Nikolenko, S. Pham, A. D. Prjibelski, A. V. Pyshkin, A. V. Sirotkin, N. Vyahhi, G. Tesler, M. A. Alekseyev, and P. A. Pevzner. 2012. SPAdes: A new genome assembly algorithm and its applications to single-cell sequencing. J. Comput. Biol. 19:455-477. https://doi.org/10.1089/cmb.2012.0021.

Bolaños, C. A. D., C. L. Paula, S. T. Guerra, M. M. J. Franco, and M. G. Ribeiro. 2017. Diagnosis of mycobacteria in bovine milk: An overview. Rev. Inst. Med. Trop. São Paulo 59:e40. https://doi.org/ 10.1590/s1678-9946201759040.

Botsaris, G., I. Slana, M. Liapi, C. Dodd, C. Economides, C. Rees, and I. Pavlik. 2010. Rapid detection methods for viable Mycobacterium avium subspecies paratuberculosis in milk and cheese. Int. J. Food Microbiol. 141(Suppl. 1):S87-S90. https://doi.org/10.1016/j .ijfoodmicro.2010.03.016.

Brode, S. K., A. Marchand-Austin, F. B. Jamieson, and T. K. Marras. 2017. Pulmonary versus nonpulmonary nontuberculous mycobacteria, Ontario, Canada. Emerg. Infect. Dis. 23:1898-1901. https:// doi.org/10.3201/eid2311.170959.

Claeys, T. A., and R. T. Robinson. 2018. The many lives of nontuberculous mycobacteria. J. Bacteriol. 200:e00739-17. https://doi.org/ 10.1128/JB.00739-17.
Costard, S., L. Espejo, H. Groenendaal, and F. J. Zagmutt. 2017. Outbreak-related disease burden associated with consumption of unpasteurized cow's milk and cheese, United States, 2009-2014. Emerg. Infect. Dis. 23:957-964. https://doi.org/10.3201/eid2306 .151603 .

Dundee, L., I. R. Grant, H. J. Ball, and M. T. Rowe. 2001. Comparative evaluation of four decontamination protocols for the isolation of Mycobacterium avium ssp. paratuberculosis from milk. Lett. Appl. Microbiol. 33:173-177. https://doi.org/10.1046/j.1472-765x .2001.00979.x

Franco, M. M., A. C. Paes, M. G. Ribeiro, J. C. de Figueiredo Pantoja, A. C. Santos, M. Miyata, C. Q. Leite, R. G. Motta, and F. J. Listoni. 2013. Occurrence of mycobacteria in bovine milk samples from both individual and collective bulk tanks at farms and informal markets in the southeast region of Sao Paulo, Brazil. BMC Vet. Res. 9:85. https://doi.org/10.1186/1746-6148-9-85.

Ghosh, P., C. Hsu, E. J. Alyamani, M. M. Shehata, M. A. Al-Dubaib, A. Al-Naeem, M. Hashad, O. M. Mahmoud, K. B. Alharbi, K. Al-Busadah, A. M. Al-Swailem, and A. M. Talaat. 2012. Genomewide analysis of the emerging infection with Mycobacterium avium subspecies paratuberculosis in the Arabian camels (Camelus dromedarius). PLoS One 7:e31947. https://doi.org/10.1371/journal.pone .0031947 .

Gopinath, K., and S. Singh. 2010. Non-tuberculous mycobacteria in TB-endemic countries: Are we neglecting the danger? PLoS Negl. Trop. Dis. 4:e615. https://doi.org/10.1371/journal.pntd.0000615.

Grant, I. R., H. J. Ball, and M. T. Rowe. 2002. Incidence of Mycobacterium paratuberculosis in bulk raw and commercially pasteurized cows' milk from approved dairy processing establishments in the United Kingdom. Appl. Environ. Microbiol. 68:2428-2435. https:/ /doi.org/10.1128/AEM.68.5.2428-2435.2002.

Honda, J. R., R. Virdi, and E. D. Chan. 2018. Global environmental nontuberculous mycobacteria and their contemporaneous manmade and natural niches. Front. Microbiol. 9:2029. https://doi .org/10.3389/fmicb.2018.02029.

Hsu, C. Y., C. W. Wu, and A. M. Talaat. 2011. Genome-wide sequence variation among Mycobacterium avium subspecies paratuberculosis isolates: A better understanding of Johne's disease transmission dynamics. Front. Microbiol. 2:236. https://doi.org/10.3389/fmicb .2011 .00236 .

Jordão Junior, C. M., F. C. M. Lopes, S. David, A. Farache Filho, and C. Q. F. Leite. 2009. Detection of nontuberculous mycobacteria from water buffalo raw milk in Brazil. Food Microbiol. 26:658-661. https://doi.org/10.1016/j.fm.2009.04.005.

Kazwala, R. R., C. J. Daborn, L. J. Kusiluka, S. F. Jiwa, J. M. Sharp, and D. M. Kambarage. 1998. Isolation of Mycobacterium species from raw milk of pastoral cattle of the Southern Highlands of Tanzania. Trop. Anim. Health Prod. 30:233-239. https://doi.org/ 10.1023/A:1005075112393.

Kendall, B. A., and K. L. Winthrop. 2013. Update on the epidemiology of pulmonary nontuberculous mycobacterial infections. Semin. Respir. Crit. Care Med. 34:87-94. https://doi.org/10.1055/s-0033 $-1333567$.

Konuk, M., E. Korcan, S. Dulgerbaki, and M. Altindis. 2007. Isolation and identification of Mycobacteria from raw milk samples in Afyonkarahisar district of Turkey. Int. J. Food Microbiol. 115:343347. https://doi.org/10.1016/j.ijfoodmicro.2006.12.019.

Kumar, S., G. Stecher, and K. Tamura. 2016. MEGA7: Molecular Evolutionary Genetics Analysis version 7.0 for bigger datasets. Mol. Biol. Evol. 33:1870-1874. https://doi.org/10.1093/molbev/ msw054.

Lai, C. C., C. K. Tan, C. H. Chou, H. L. Hsu, C. H. Liao, Y. T. Huang, P. C. Yang, K. T. Luh, and P. R. Hsueh. 2010. Increasing incidence of nontuberculous mycobacteria, Taiwan, 2000-2008. Emerg. Infect. Dis. 16:294-296. https://doi.org/10.3201/eid1602.090675.

Leite, C. Q., I. S. Anno, S. R. Leite, E. Roxo, G. P. Morlock, and R. C. Cooksey. 2003. Isolation and identification of mycobacteria from livestock specimens and milk obtained in Brazil. Mem. Inst. Oswaldo Cruz 98:319-323. https://doi.org/10.1590/S0074 -02762003000300005 . 
McKee, R., I. R. Grant, M. T. Rowe, H. G. Buckley, J. F. Buckley, and S. Fanning. 2002. Examination of in-line filters to detect Mycobacterium avium ssp. paratuberculosis infection at farm level. Pages 258-260 in Proc. 7th Int. Colloq. Paratuberculosis. R. A. Juste, M. V. Geijo, and J. M. Garrido, ed. International Association for Paratuberculosis Inc., Madison, WI.

Moore, J. E., M. E. Kruijshaar, L. P. Ormerod, F. Drobniewski, and I. Abubakar. 2010. Increasing reports of non-tuberculous mycobacteria in England, Wales and Northern Ireland, 1995-2006. BMC Public Health 10:612. https://doi.org/10.1186/1471-2458-10-612.

NCBI Resource Coordinators. 2016. Database resources of the National Center for Biotechnology Information. Nucleic Acids Res. 44(D1):D7-D19. https://doi.org/10.1093/nar/gkv1290.

Nishiuchi, Y., T. Iwamoto, and F. Maruyama. 2017. Infection sources of a common non-tuberculous mycobacterial pathogen, Mycobacterium avium complex. Front. Med. (Lausanne) 4:27. https://doi .org/10.3389/fmed.2017.00027.

Panagiotou, M., A. I. Papaioannou, K. Kostikas, M. Paraskeua, E. Velentza, M. Kanellopoulou, V. Filaditaki, and N. Karagiannidis. 2014. The epidemiology of pulmonary nontuberculous mycobacteria: Data from a general hospital in Athens, Greece, 2007-2013. Pulm. Med. 2014:894976. https://doi.org/10.1155/2014/894976.

Sevilla, I. A., E. Molina, M. Tello, N. Elguezabal, R. A. Juste, and J. M. Garrido. 2017. Detection of mycobacteria by culture and DNA-based methods in animal-derived food products purchased at Spanish supermarkets. Front. Microbiol. 8:1030. https://doi.org/ 10.3389/fmicb.2017.01030.

Sgarioni, S. A., R. D. Hirata, M. H. Hirata, C. Q. Leite, K. A. de Prince, S. R. de Andrade Leite, D. V. Filho, V. L. Siqueira, K. R. Caleffi-Ferracioli, and R. F. Cardoso. 2014. Occurrence of Mycobacterium bovis and non-tuberculous mycobacteria (NTM) in raw and pasteurized milk in the northwestern region of Parana, Brazil. Braz. J. Microbiol. 45:707-711. https://doi.org/10.1590/S1517 $-83822014000200046$

Shankar, H., S. V. Singh, P. K. Singh, A. V. Singh, J. S. Sohal, and R. J. Greenstein. 2010. Presence, characterization, and genotype profiles of Mycobacterium avium subspecies paratuberculosis from unpasteurized individual and pooled milk, commercial pasteurized milk, and milk products in India by culture, PCR, and PCRREA methods. Int. J. Infect. Dis. 14:e121-e126. https://doi.org/ 10.1016/j.ijid.2009.03.031.

Shitaye, J. E., A. Horvathova, L. Bartosova, M. Moravkova, M. Kaevska, N. Donnelly, and I. Pavlik. 2009. Distribution of nontuberculosis mycobacteria in environmental samples from a slaughterhouse and in raw and processed meats. Czech J. Food Sci. 27:194-202. https://doi.org/10.17221/120/2008-CJFS.

Shojaei, H., P. Heidarieh, A. Hashemi, M. M. Feizabadi, and A. Daei Naser. 2011. Species identification of neglected nontuberculous mycobacteria in a developing country. Jpn. J. Infect. Dis. 64:265-271.
Slana, I., P. Kralik, A. Kralova, V. Babak, and I. Pavlik. 2012. Short communication: Examination of milk filters by real-time PCR as a herd-level indicator of the presence of Mycobacterium avium subspecies paratuberculosis in dairy herds. J. Dairy Sci. 95:1162-1165. https://doi.org/10.3168/jds.2011-4658.

Slana, I., P. Kralik, A. Kralova, and I. Pavlik. 2008. On-farm spread of Mycobacterium avium ssp. paratuberculosis in raw milk studied by IS900 and F57 competitive real time quantitative PCR and culture examination. Int. J. Food Microbiol. 128:250-257. https://doi.org/ 10.1016/j.ijfoodmicro.2008.08.013.

Talaat, A. M., R. Reimschuessel, and M. Trucksis. 1997. Identification of mycobacteria infecting fish to the species level using polymerase chain reaction and restriction enzyme analysis. Vet. Microbiol 58:229-237. https://doi.org/10.1016/S0378-1135(97)00120-X.

Tamura, K., G. Stecher, D. Peterson, A. Filipski, and S. Kumar. 2013. MEGA6: Molecular Evolutionary Genetics Analysis Version 6.0. Mol. Biol. Evol. 30:2725-2729. https://doi.org/10.1093/molbev/ mst197.

Treangen, T. J., B. D. Ondov, S. Koren, and A. M. Phillippy. 2014. The Harvest suite for rapid core-genome alignment and visualization of thousands of intraspecific microbial genomes. Genome Biol. 15:524. https://doi.org/10.1186/s13059-014-0524-x.

Wisconsin Department of Health Services. 2019. Tuberculosis statistics. Accessed April 2019. https://www.dhs.wisconsin.gov/tb/data .htm.

Wolinsky, E. 1979. Nontuberculous mycobacteria and associated diseases. Am. Rev. Respir. Dis. 119:107-159.

Yoder, S., C. Argueta, A. Holtzman, T. Aronson, O. G. Berlin, P. Tomasek, N. Glover, S. Froman, and G. Stelma Jr.. 1999. PCR comparison of Mycobacterium avium isolates obtained from patients and foods. Appl. Environ. Microbiol. 65:2650-2653. https:// doi.org/10.1128/AEM.65.6.2650-2653.1999.

Yoo, J. W., K. W. Jo, M. N. Kim, S. D. Lee, W. S. Kim, D. S. Kim, and T. S. Shim. 2012. Increasing trend of isolation of non-tuberculous mycobacteria in a tertiary university hospital in South Korea. Tuberc. Respir. Dis. (Seoul) 72:409-415. https://doi.org/10.4046/ trd.2012.72.5.409.

\section{ORCIDS}

Zeinab I. Ali @ https://orcid.org/0000-0003-0938-0604 Mostafa Hanafy ๑ https://orcid.org/0000-0002-4776-0352 Adel M. Talaat ๑ https://orcid.org/0000-0003-3305-7903 